\title{
Olfactory Neuroblastoma From Ala Of Nose- An Unusual Presentation
}

\author{
Dr. Vinod Kandakure \\ M.S. (ENT) Assistant Professor, Government Medical College, Latur. Maharashtra, India.
}

\begin{abstract}
Esthesioneuroblastoma(Olfactory neuroblastoma) is a rare malignant neoplasm arising from basal cells within the olfactory epithelium. Its clinnical course is unpredictable with variable correlation between microscopic features and biological behaviour.[1] We report a very indolent case of olfactory neuroblastoma presented only with nasal obstruction due to unilateral mass at the anterolateral aspect of nasal ala of left side. The mass excised via lateral rhinotomy approach and diagnosis of olfactory neuroblastoma was established by histopathology.
\end{abstract}

Key Words- Esthioneuroblastoma, olfactory neuroblastoma, computed tomography.

\section{Introduction}

Olfactory neuroblastoma, also called esthesioneuroblastoma, is a very malignant tumor believed to originate in olfactory cells most commonly presented as unilateral polypoid nasal masses often causing nasal obstruction and epistaxis. It has a predilection for invading surrounding regions, such as the paranasal sinuses, orbits skull base and can cause loss of smell, taste, and vision, as well as facial disfigurement in advanced cases.[2]Olfactory neuroblastoma is a neuroendocrine tumour capable of causing paraneoplastic syndrome by secreting peptides.[3]

Olfactory neuroblastoma often responds well to radiation therapy, but the tumor has a high tendency to recur after excision. Despite its name, olfactory neuroblastoma is considered distinct from other neuroblastomas, because it does not originate in the sympathetic nervous system.[4]

\section{Case History}

A 12-year-old male child presented with a 15 days history of left nasal obstruction due to slightly painful swelling in left nostril, obstruction was persistent, non progressive and not relieved with medication. On palpation, $3 \times 3 \mathrm{~cm}$ sized single, firm, mildly tender mass arising from lateral wall, pushing the left ala laterally was seen. (Fig.1). No bleeding on probing was seen. Cold spatula test shows decreased fogging on left side. He had no history of associated epistaxis, headaches, diplopia, or sensory changes. Physical examination revealed a mass in the left nasal cavity arising from anterolateral aspect of left nostril. Examination of the right nasal cavity was normal, as was the remainder of the head and neck examination. Computed tomography (CT) with contrast showed a heterogeneously enhancing oval shaped mass within the left nasal cavity arising from anterolateral and nasolabial aspect. There is no evidence of erosion or destruction of adjacent bone due to mass. (Fig.2)

Surgery: Mass exposed by lateral rhinotomy incision. It is then dissected and delineated from all sides. Around $3 \times 3 \mathrm{~cm}$ rounded pale pinkish tumor removed completely. (Fig.3) Suturing was done in layers.

Histopathology : A tumor composed of cells arranged in irregular circumscribed nests of cells separated by stroma. The cells have high N/C ratio, indistinct cell borders, round vesicular nuclei with clumped chromatin and scanty cytoplasm. The nuclei are surrounded by neurofibrillary material. Pseudorosettes (Homer Wright Rosette) characterized by grouping of cells in circumscribed fashion around neurofibrillary material but without defining basement membrane are seen. (Fig.4) On immunohistochemistry, tumor stain positive for S-100 protein.

A diagnosis of a grade I esthesioneuroblastoma was made on biopsy \& histopathology of the nasal mass.

\section{Discussion}

Olfactory neuroblastomas are solid nasal cavity masses that can erode into nearby osseous structures such as the orbital plate of the ethmoid bone, cribriform plate, and fovea ethmoidalis. The most common finding on physical examination is a unilateral, broad-based, polypoid, friable, and gray-to-red nasal mass. The two most common symptoms presented were unilateral nasal obstruction and epistaxis $[2,5,6]$ 
The disease has a bimodal age distribution; it peaks first between 10 and 20 years and then between 50 and 60 years.[7] The distribution is not affected by sex race.

The symptoms of esthesioneuroblastoma (ENB) can be classified into nasal, neurologic, oral, facial, cervical, and ophthalmologic and are as follows:

- Nasal - Obstruction (70\%), epistaxis (46\%), discharge, unilateral polyp, anosmia

- Neurologic - Headache, nausea

- Oral (rare) - Mobile tooth, nonhealing tooth-extraction site, ill-fitting dental prostheses, ulceration

- Facial (rare) - Swelling, pain, anesthesia, trismus

- Cervical - Mass

- Ophthalmologic - proptosis, extraocular movement paralysis, and blindness,

The average delay between the appearance of the initial symptom and the diagnosis is 6 months, but diagnosis is delayed for years in some cases.

Nasal examination, particularly if aided by endoscopy, reveals a reddish-gray tumor arising in the upper nasal fossa, which bleeds easily with instrumentation. Although this aspect is strictly different from the white, glistening appearance of benign nasal polyps, little differentiates esthesioneuroblastoma (ENB) from other nasal malignancies. Late findings may include signs related to extensive disease such as orbital, cranial, and cervical involvement.

No clear etiologic agent or exposure has been documented in humans; however, a single case of occupational exposure has been reported in a woodworker.[8] Esthesioneuroblastoma (ENB) can be consistently induced by nitrosamine compounds in rodents. In cats with spontaneous ENB or in transgenic mice developing $\mathrm{ENB}$, type $\mathrm{C}$ retroviral particles have been demonstrated and classified as feline and murine leukemia virus, respectively. The role of retrovirus sequences in human ENB remains to be evaluated.

Differential diagnosis can be non-Hodgkins lymphoma, malignant melanoma, plasmacytoma.

No specific lab studies confirm the diagnosis of esthesioneuroblastoma (ENB). Because surgery often isA direct coronal fine-cut CT scan $(3 \mathrm{~mm})$ is the initial radiologic study of choice. and is seen as a homogeneous soft tissue mass with uniform and moderate contrast enhancement, CT images are essential for correct staging and should be evaluated carefully for erosion of the lamina papyracea, cribriform plate, and fovea ethmoidalis specifically.Obstruction of the sinus-draining ostia results in an accumulation of nasal secretions, which tend to be difficult to differentiate from tumor tissue when viewed on CT scan.An unusual but characteristic imaging feature of ENBs is the presence of cysts at the tumor-brain interface. MRI often is necessary to better delineate sinonasal and intraorbital extension or an intracerebral extension.Using MRI, ENB appears as hypointense to gray matter on T1-weighted images and isointense or hyperintense to gray matter on T2-weighted images.Excision biopsy specimen should be deferred until completion of the radiologic studies to avoid swelling effects on accurate imaging and the inadvertent biopsy of other nasal tumors of neurogenic origin.Biopsy and endoscopy should be performed under general anesthesia. The specimen should be sent for regular staining, as well as for immunohistochemistry and possibly electron microscopy. The hallmark of welldifferentiated ENBs is arrangements of cells into rosettes or pseudorosettes (sheets and clusters). True rosettes (Flexner-Wintersteiner rosettes) refer to a ring of columnar cells circumscribing a central oval-to-round space, which appears clear on traditional pathologic sections. Pseudorosettes (Homer-Wright rosettes) are characterized by a looser arrangement and the presence of fibrillary material within the lumen.

On immunohistochemistry Esthesioneuroblastomas stain positive for S-100 protein and/or neuronspecific enolase, while the stain usually is negative for cytokeratin, desmin, vimentin, actin, glial fibrillary acidic protein, UMB 45, and the common leukocytic antigen. For difficult cases, electron microscopy can be useful. Common features are small, round neuroepithelial cells arranged in rosette or pseudorosette patterns, separated by fibrous elements. Rosettes consist of a central space ringed by columnar cells with radially oriented nuclei. ENB can be graded histologically by the Hyams system, which is based on the preservation of lobular architecture, mitotic index, nuclear polymorphism, and the presence of fibrillary matrix, rosettes, and necrosis. ${ }^{[9]}$ The Hyams system is based on 4 grades, which are described in Table 1, below. 
Table1. Histopathologic Grading According to Hyams ${ }^{[9]}$

\begin{tabular}{|lllllll|}
\hline \hline Grade & $\begin{array}{l}\text { Lobular Architecture } \\
\text { Preservation }\end{array}$ & $\begin{array}{l}\text { Mitotic } \\
\text { Index }\end{array}$ & $\begin{array}{l}\text { Nuclear } \\
\text { Polymorphism }\end{array}$ & $\begin{array}{l}\text { Fibrillary } \\
\text { Matrix }\end{array}$ & Rosettes & Necrosis \\
I & + & Zero & None & Prominent & $\begin{array}{l}\text { HW } \\
\text { rosettes }\end{array}$ & None \\
II & + & Low & Low & Present & $\begin{array}{l}\text { HW } \\
\text { rosettes }\end{array}$ & None \\
III & + t- & Moderate & Moderate & Low & $\begin{array}{l}\text { FW } \\
\text { rosettes }\end{array}$ & Rare \\
IV & High & High & Absent & None & Frequent \\
\hline
\end{tabular}

Tumour staging by Kadish and TNM staging......From a limited series of 17 patients, Kadish et al were the first to propose a staging classification for esthesioneuroblastoma (ENB). ${ }^{[10]}$ ENBs were divided into 3 categories: groups A, B, and C. Group A is limited to tumors of the nasal fossa; in group B, extension is to the paranasal sinuses; and group $\mathrm{C}$ is defined as extension beyond the paranasal sinuses and nasal cavity.

In 1992, Dulguerov and Calceterra proposed a classification based on the tumor, node, metastasis (TNM) system, which is predicated on CT and MRI findings that can be identified before treatment. ${ }^{[1]}$ Although this classification system has gained popularity, attempts have been made to further modify the Kadish system for ENB.

- $\quad$ TNM system, where $\mathrm{T}=$ tumor, $\mathrm{N}=$ node, and $\mathrm{M}=$ metastasis

○ T1 - Tumor involving the nasal cavity and/or paranasal sinuses (excluding sphenoid), sparing the most superior ethmoidal cells

- T2 - Tumor involving the nasal cavity and/or paranasal sinuses (including the sphenoid), with Sextension to or erosion of the cribriform plate

○ T3 - Tumor extending into the orbit or protruding into the anterior cranial fossa, without dural invasion.

- T4 - Tumor involving the brain

- N0 - No cervical lymph node metastasis

○ $\quad$ 1 - Any form of cervical lymph node metastasis

- M0 - No metastasis

○ M1 - Distant metastases present

TREATMENT: The literature gives little support to single-modality treatments; few studies advocate either surgery or radiation alone. Dulguerov's 2001 meta-analysis clearly showed lower recurrence rates for the combination of surgery and radiotherapy. ${ }^{[12]}$ Some institutions advocate surgery alone for Kadish stage A tumors, whereas most suggest adjuvant radiotherapy for these lesions.A few studies advocate neoadjuvant chemotherapy for Kadish C lesions.

Unlike most surgical specimens from the head and neck, specimens from the nasal cavity and paranasal sinuses, even en bloc, are difficult to orient, and surgical margins are difficult to analyze. Because one can rarely be completely confident of the adequacy of surgical margins, postoperative radiation to minimize the risk of local recurrence seems justified in almost all patients.

Timing of surgery and radiation in combined therap Most institutions favor surgery as the first treatment modality, followed by postoperative irradiation.

PROGNOSIS: Prognostic survival factors that emerged in the meta-analysis by Dulguerov et al include lower Hyams histopathologic grade (56\% for grades I-II vs. $25 \%$ for grades III-IV) and absence of neck lymph node metastasis (64\% for N0 vs. $29 \%$ for $\mathrm{N}+)^{[12]}$

Other possible but unconfirmed prognostic factors include a low proliferative index, the completeness of the surgical resection, and tumor shrinkage after induction chemotherapy.

In recent series, the 5-year patient survival rates have varied from 50-80\%, with the majority of large studies indicating patient survival rates of higher than $70 \%$. In the 2001 meta-analysis by Dulguerov et al, the average 5-year survival was $45 \pm 22 \%$ (range, $0-86 \%)^{[12]}$ 


\section{Conclusion}

Olfactory neuroblastoma can either be extremely aggressive or relatively indolent. After imaging definitive diagnosis is possible with excision biopsy and confirmation by immunohistochemistry. From this case we conclude that clinical diagnosis is not possible in early stages especially if it originates from unusual sites.

\section{References}

[1] Scott - Brown's Otolaryngology,Head and Neck Surgery, $7^{\text {th }}$ ed.,vol. 3 .

[2] Cummings C W. Cummings Otolaryngology: Head and Neck Surgery. 4th ed. St. Louis: Mosby Inc; 2005. p. 3915

[3] Min KW. Usefulness of electron microscopy in the diagnosis of "small" round cell tumors of the sinonasal region. Ultrastruct Pathol. Sep-Oct 1995;19(5):347-63. [Medline].

[4] Written by Niki Foster, edited by Andrew Jones.Last modified date 8 may 2012

[5] Morita A, Ebersold M J, Olsen K D, et al. Esthesioneuroblastoma: prognosis and management.Neurosurgery. 1993;32:706715. [PubMed]

[6] Simon J H, Zehn W, McCulloch T M, et al. Esthesioneuroblastoma: the University of Iowa experience 19781998. Laryngoscope. 2001;111:488-493. [PubMed

[7] Broich G, Pagliari A, Ottaviani F. Esthesioneuroblastoma: a general review of the cases published since the discovery of the tumour in 1924. Anticancer Res. 1997;17:2683-2706. [PubMed

[8] Hyams VJ, Batsakis JG, Michaels L. Olfactory neuroblastoma. In: Tumors of the Upper Respiratory Tract and Ear, Atlas of Tumor Pathology. Vol 25. Washington DC: Armed Forces Institute Press; 1988: . 240-8.

[9] Fitzek MM, Thornton AF, Varvares M. Neuroendocrine tumors of the sinonasal tract. Results of a prospective study incorporating chemotherapy, surgery.

[10] Kadish S, Goodman M, Wang CC. Olfactory neuroblastoma. A clinical analysis of 17 cases. Cancer. Mar 1976;37(3):15716. [Medline].

[11] Dulguerov P, Calcaterra T. Esthesioneuroblastoma: the UCLA experience 1970-1990. Laryngoscope. Aug 1992;102(8):8439. [Medline].

[12] Dulguerov P, Allal AS, Calcaterra TC. Esthesioneuroblastoma: a meta-analysis and review. Lancet Oncol. Nov 2001;2(11):68390. [Medline].

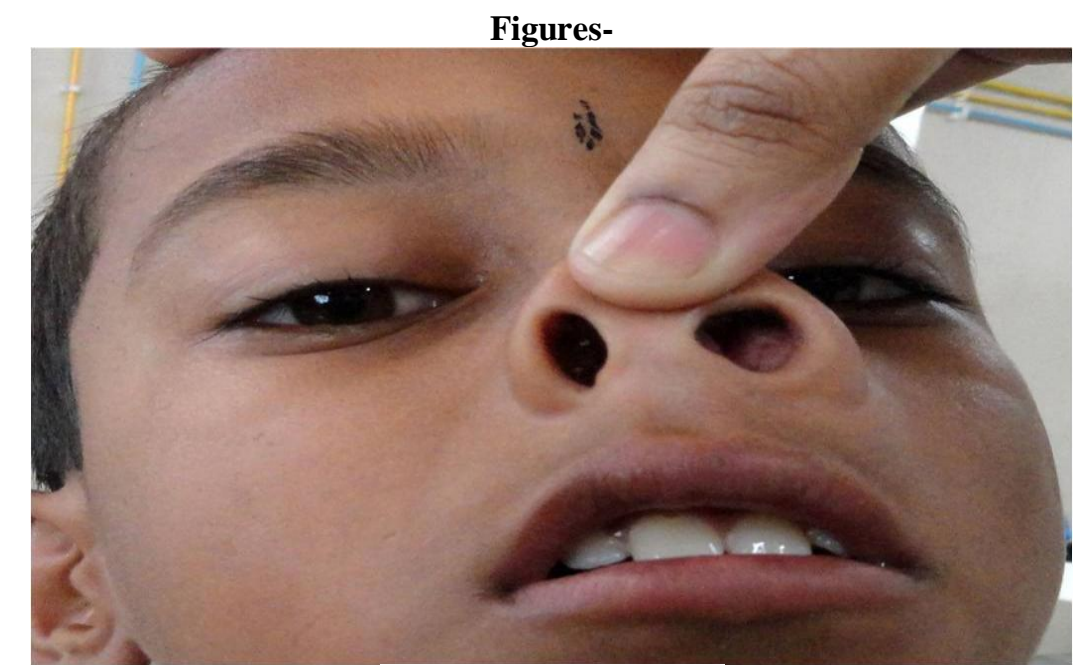

Fig.1- Left Nostril mass.

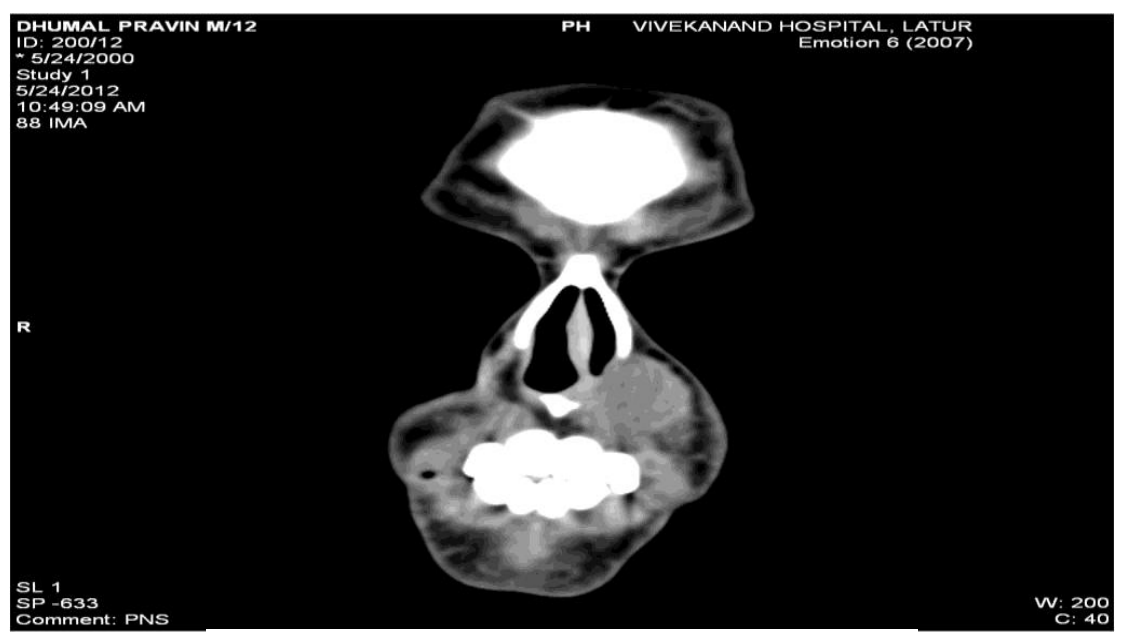

Fig.2- CT PNS Coronal showing left nostril tumor. 


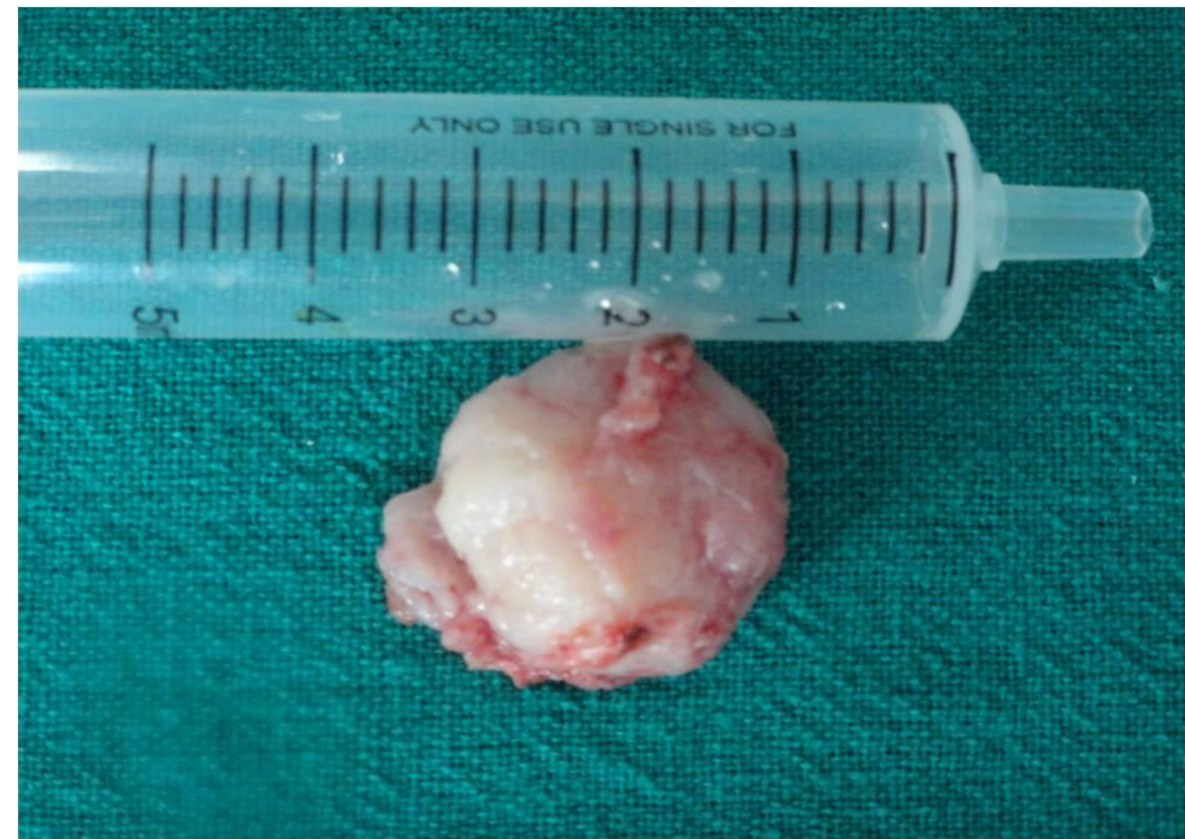

Fig.3- Excised tumor.

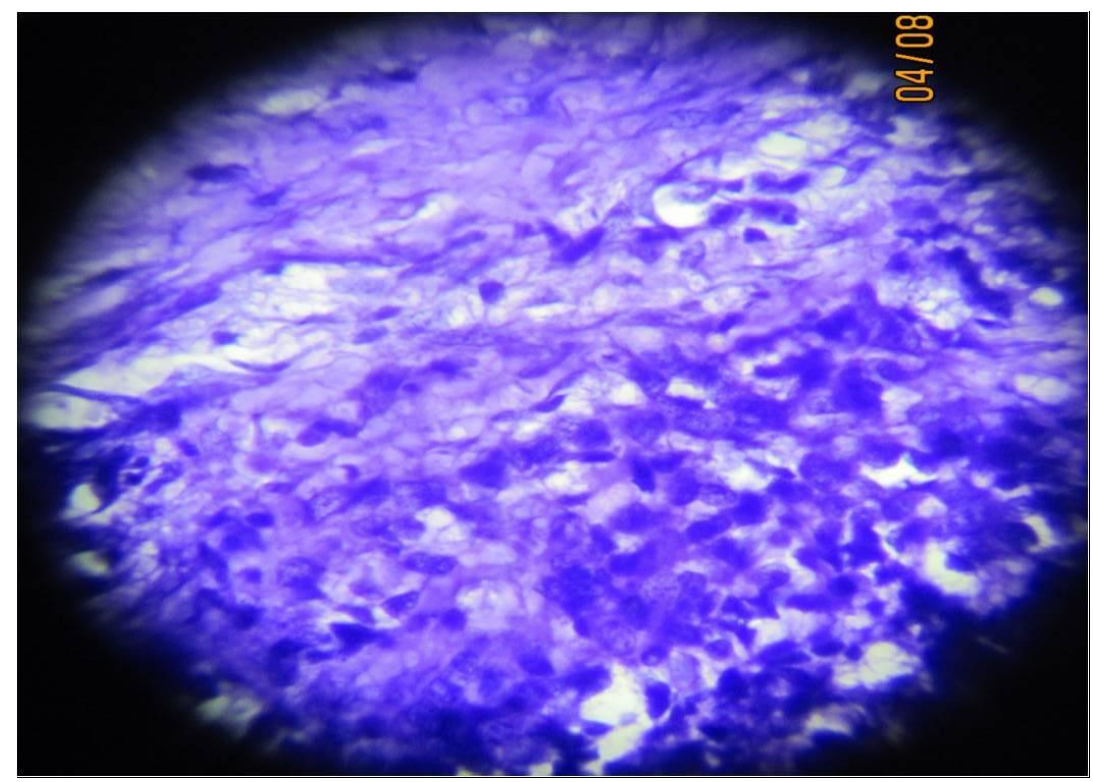

Fig.4- Histopathology slide. 